\title{
Pre-operative education importance on muscle strength and the knee joint condition results for patients after anterior cruciate ligament reconstruction: a pilot study
}

\author{
Aistė Vainiūtė ${ }^{1},{ }^{3}$, Vytautas Tutkus ${ }^{2},{ }^{4}$, leva Eglè Jamontaitė ${ }^{1,3}$ \\ ${ }^{1}$ Vilnius University, Faculty of Medicine, The Department of Rehabilitation, Physical and Sports \\ Medicine \\ ${ }^{2}$ Vilnius University, Faculty of Medicine, The Department of Anatomy, Histology and \\ Anthropology \\ ${ }^{3}$ Vilnius University Hospital Santaros Clinics, Rehabilitation, Physical and Sports Medicine Centre \\ ${ }^{4}$ Vilnius University Hospital Santaros Clinics, Plastic and Reconstructive surgery centre
}

Introduction The anterior cruciate ligament (ACL) is an important stabilizing ligament of the knee. An annual incidence in general population is approximately $1 \mathrm{ACL}$ rupture in 3500 inhabitants. Usually insufficient or noncorrect rehabilitation makes a negative effect for the restoration of functional status for patients who undergo ACL surgery. For this reason, early pre-operative education (POE) of rehabilitation principles and physiotherapy methods is important in achieving the desired postoperative results.

Purpose To evaluate the POE importance for muscle isometric strength (MIS) restoration and the knee joint condition (KJC) improvement on patients who undergone ACL reconstruction.

Method The 21 patient $(32.9 \pm 9.99$ years) involved in this study was divided in two groups: experimental group (EG) (consisting of 11 participants), in this group the extensive POE was applied, a home-based program with explanations was provided, and a control group (CG) (consisting of 10 participants) that received a brief verbal education and was provided with the customized memo. The Lysholm questionnaire was used to evaluate the $\mathrm{KJC}$ and the Lafayette manual dynamometer for the MIS evaluation of the calf flexors (CF) and extensors (CE). Measurements were made before reconstruction, one and three month after surgery in both groups.

Results A month after the surgery, the average scores of the questionnaire showed that the $\mathrm{KJC}$ of $\mathrm{EG}$ remained satisfactory and in the CG was worst. After three months EG evaluated its KJC 14.6 points $(p<0.05)$ better than $C G$ and 8.18 points $(p<0.05)$ better than before the reconstruction (Figure 1). After a month the MIS of the CE was $82.65 \mathrm{~N}$ $(p<0.05)$ and of the CF muscle was $18.32 \mathrm{~N}(p>0.05)$ higher in the $E G$ than in $C G$. After three months $E G$ was able to develop a higher CE-61.55N $(p<0.05)$ and CF-33.49N

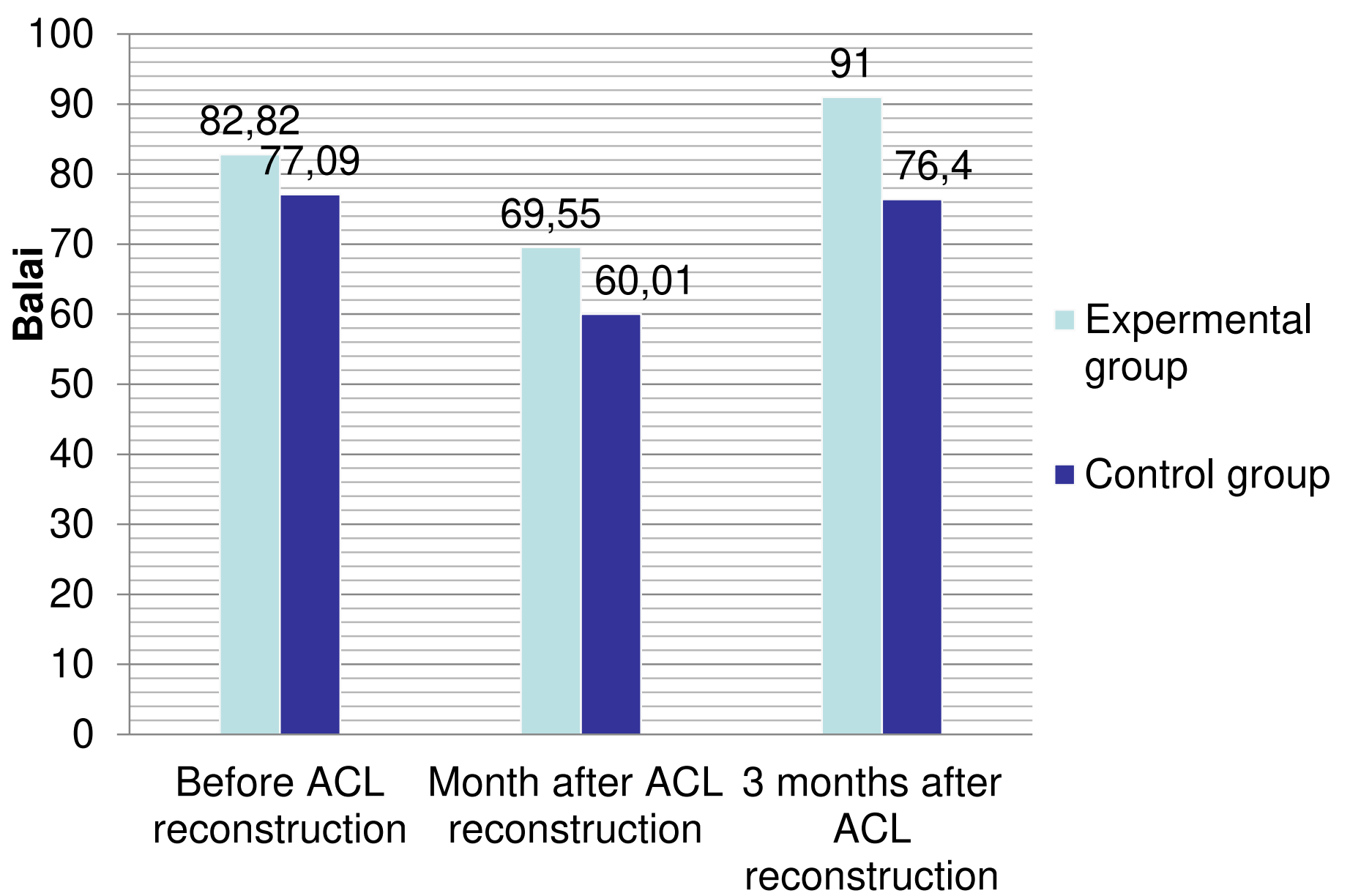

Figure 1. "Lysholm" questionnaire results.

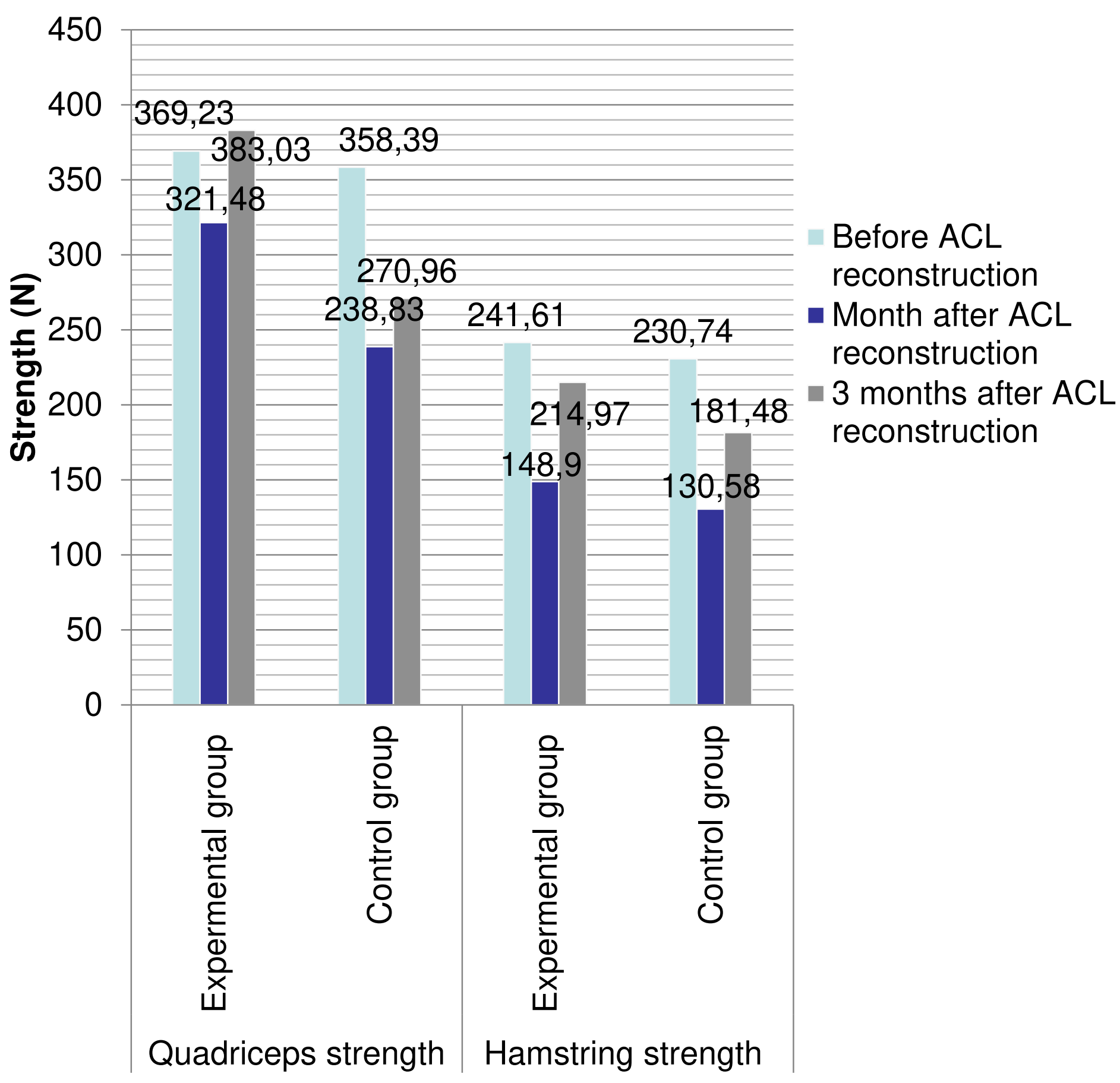

Figure 2. Results of isometric muscle strength of the operated leg. ( $p>0.05)$ MIS than CG (Figure 2).

Conclusions Pre-operative education gives better muscle isometric strength and the knee joint condition recovery results in early postoperative period.

\section{Sources}

1. Grindem H, Risberg MA, Eitzen I. Two factors that may underpin outstanding outcomes after ACL rehabilitation. British journal of sports medicine. 2015;49:1495.

2. Malempati Ch, Jurjans J, Noehren B, Ireland ML, Johnson DL. Current Rehabilitation Concepts for Anterior Cruciate Ligament Surgery 